\title{
Two-electron processes in multiple ionization under strong soft-x-ray radiation
}

\author{
M. Ilchen, ${ }^{1,2}$ T. Mazza, ${ }^{1}$ E. T. Karamatskos,${ }^{3, *}$ D. Markellos, ${ }^{3}$ S. Bakhtiarzadeh, ${ }^{1}$ A. J. Rafipoor, ${ }^{1}$ T. J. Kelly, ${ }^{4}$ N. Walsh, \\ J. T. Costello, ${ }^{4}$ P. O' Keeffe, ${ }^{5}$ N. Gerken, ${ }^{6}$ M. Martins, ${ }^{6}$ P. Lambropoulos, ${ }^{3}$ and M. Meyer ${ }^{1}$ \\ ${ }^{1}$ European XFEL GmbH, Albert-Einstein-Ring 19, D-22761 Hamburg, Germany \\ ${ }^{2}$ Stanford PULSE Institute, SLAC National Accelerator Laboratory, Menlo Park, California 94025, USA \\ ${ }^{3}$ IESL-FORTH, P.O. Box 1527, and Physics Department, University of Crete, Heraklion, Crete 71110, Greece \\ ${ }^{4}$ School of Physical Sciences and NCPST, Dublin City University, Dublin 9, Ireland \\ ${ }^{5}$ CNR Istituto di Struttura della Materia, CP10, I-00016 Monterotondo Scalo, Italy \\ ${ }^{6}$ Universität Hamburg, Institut für Experimentalphysik, Luruper Chaussee 149, 22761 Hamburg, Germany
}

(Received 24 May 2016; published 18 July 2016)

\begin{abstract}
In a combined experimental and theoretical study we have investigated the ionization of atomic argon upon irradiation with intense soft-x-ray pulses of $105 \mathrm{eV}$ photon energy from the free-electron laser FLASH. The measured ion yields show charge states up to $\mathrm{Ar}^{7+}$. The comparison with the theoretical study of the underlying photoionization dynamics highlights the importance of excited states in general and of processes governed by electron correlation in particular, namely, ionization with excitation and shake-off, processes usually inaccessible by measurements of ionic yields only. The $\mathrm{Ar}^{7+}$ yield shows a clear deviation from the predictions of the commonly used model of sequential ionization via single-electron processes and the observed signal can only be explained by taking into account the full multiplet structure of the involved configurations and by inclusion of two-electron processes. The competing process of two-photon ionization from the ground state of $\mathrm{Ar}^{6+}$ is calculated to be orders of magnitude smaller.
\end{abstract}

DOI: 10.1103/PhysRevA.94.013413

\section{INTRODUCTION}

Photoionization of any multi-electron system entails the production of ions in one or more excited states. This is usually referred to as ionization plus excitation or shake-up, if the photon energy is sufficiently large for the excess energy (beyond the first ionization threshold) to reach one or more excited ionic states. In the case where the photon energy is high enough to reach the double-ionization threshold, shake-off processes become also possible. In single-photon ionization, these two-electron $(2 e)$ processes can occur only through electron correlation. As such, they are relatively weak, with the value of the corresponding cross section typically in the range of a few percent of the dominant one-electron process. The process has been studied both theoretically (Refs. [1-3] and references therein) and experimentally (Refs. [3-5] and references therein) in a variety of systems, as it demonstrates in a direct way the limits of, and deviations from, the simple one-electron pictures. The detailed understanding of the importance of such electron correlation is of fundamental importance for any photoionization process, i.e., in atoms as well as in molecules, clusters, or solids, and for the evaluation of the accuracy of sophisticated theoretical models.

Many of the recent experiments at the new free-electron laser (FEL) sources, concerned with the investigation of multiple ionization under intense short wavelength radiation, are based on measurements of ion yields [6-9]. The rather simple appearance of an ion spectrum provides valuable insights into the complex processes and pathways, involving the intricate interplay between single- and multiphoton ionization [10] and,

\footnotetext{
*Present address: Center for Free-Electron Laser Science (CFEL), Deutsches Elektronen-Synchrotron DESY, Notkestrasse 85, 22607 Hamburg, Germany.
}

in the case of inner shell ionization, the subsequent relaxation via Auger decay [11-13]. However, detection of the ionic species, which consist of both ground- and excited-state ions, cannot in general provide any detailed information on the relative importance of electron correlations in the various processes leading to multiple ionization. The detection and quantification of ionization plus excitation, requires, e.g., the analysis of photoelectron energy spectra, where the excitation is observed as a satellite structure beside the main photo lines $[4,5]$ and shake-off processes cause a flat, symmetric, Ushaped background to the discrete photolines (e.g. Ref. [14]). In principle, the detection of fluorescence from the excited states of the residual ion can also serve as a probe of this process (e.g. Ref. [15]). Traditionally, these studies have been performed at synchrotron radiation facilities, which are restricted to the observation of single-photon processes and generally to neutral targets. Recently, the application of the covariance mapping technique was introduced for ionization studies at FELs enabling, e.g., the identification of shake processes in the corresponding electron spectra [16].

However, in the process of studying multiple ionization of argon under strong soft-X-ray FEL radiation of $105 \mathrm{eV}$ photon energy, we discovered strong indications of ionization plus excitation, in the relative abundance of ionic species, without any recourse to photoelectron energy spectra. Moreover, our study including the full multiplet structure of the involved electron configurations produces support for the involvement of ionization plus excitation in the ionic species up to $\mathrm{Ar}^{5+}$.

Details for the experimental procedure to record ion yields at the free-electron laser FLASH in Hamburg are given in Sec. II. The theoretical approaches used to treat the sequential ionization processes are summarized in Sec. III. The experimental results and their interpretation on the basis of the theoretical work are presented in Sec. IV, followed by a short conclusion. 


\section{EXPERIMENT}

In the present study we have investigated the ionization processes in a dilute argon gas jet upon irradiation with pulses of $105 \mathrm{eV}(11.8 \mathrm{~nm})$ photon energy from the free-electron laser in Hamburg (FLASH) [17]. The experiments were performed at the BL2 beamline using FEL pulses of about $80 \pm 20 \mathrm{fs}$ in duration, determined from the number of modes in the measured spectral distribution of the FEL radiation [18], and pulse energies of up to $40 \mu \mathrm{J}$. The actual FEL intensity was determined for each individual pulse using a gas monitor detector [19]. $\mathrm{A} \mathrm{MoB}_{4} \mathrm{C}$ multilayer mirror, with a reflection bandwidth of about $1 \mathrm{eV}$ centered at $105 \mathrm{eV}$, was used in a back-reflecting geometry to tightly focus the FEL beam to a diameter of $4 \pm 1 \mu \mathrm{m}$ in the interaction region with the effusive Ar beam coinciding with the acceptance volume of an ion time-of-flight spectrometer. By taking into account the mirror reflectivity of $40 \% \pm 1 \%$ for $105 \mathrm{eV}$ photon energy we were able to achieve irradiance levels of up to $7.2 \times 10^{14} \mathrm{~W} \mathrm{~cm}^{-2}$. The photon energy bandwidth of FLASH was measured with a variable line spacing spectrometer [17] to be $1 \%$. For recording intensity-dependent data over a wide dynamic range, a gas attenuation system was used, which eliminates the possible dependence of the interaction volume on intensity.

A conventional time-of-flight spectrometer, composed of a 4-cm-wide acceleration region around the interaction volume and a 65-cm-long drift tube, was used to separate the different charge states of the Ar ions. Typically an extraction field of $500 \mathrm{~V} / \mathrm{cm}$ was applied to ensure that all ions were collected and directed towards a multichannel plate assembly at the end of the drift tube. Possible variations in the detection efficiency for the different charge states were estimated by recording spectra with different voltage settings on the ion detector and have been taken into account in the error bars of the ion yields.

\section{THEORY}

Under the conditions of the experiment, the quantitative description of the interaction can be cast in terms of rate equations, with proper account of the pulse duration, peak intensities, and interaction volume integration, defined by the dimensions of the focused radiation source and its overlap with the atomic beam. For peak intensities below $10^{16} \mathrm{~W} \mathrm{~cm}^{-2}$ and pulse durations of $80 \mathrm{fs}$, which means hundreds of field cycles long, the use of rate equations and the notion of the cross section are well within their validity [10]. The values of the single-photon as well as the two-photon cross sections have been calculated as discussed in the Appendix. The fine structure manifolds of the $3 p$ and $3 s$ shells of the neutral atom and its ions have been accounted for in the calculation of the overall cross sections entering the sequence of the ionization channels. The complete set of the rate equations, cross sections and related discussion are provided in the Appendix.

Since above threshold ionization (ATI) processes have already been observed in other studies with similar FEL parameters [20-22], we have also included in the rate equations two ATI channels (from the neutral as well as from the first ion) with cross sections that have been calculated. Note that ATI, leading from the neutral atom to $\mathrm{Ar}^{+}$, as well as from $\mathrm{Ar}^{+}$to $\mathrm{Ar}^{2+}$, contributes on the level of $1.87 \times 10^{-4}$ and $2.44 \times 10^{-5}$ to the $\mathrm{Ar}^{+}$and $\mathrm{Ar}^{2+}$ yields, respectively. Although their contribution to the respective ionic species is extremely small, their incorporation in the calculation and comparison to the experimental data serve as an additional test of the theoretical model.

We included also shake-off processes in the calculations. The cross section for shake-off from Ar leading to $\mathrm{Ar}^{2+}$ is known through experimental as well as theoretical studies [3]. If that process is not included in the rate equations, the theoretical yield of $\mathrm{Ar}^{2+}$ is about $50 \%$ lower than the experimental data. There is also a similar process from $\mathrm{Ar}^{+}$ leading to $\mathrm{Ar}^{3+}$, with a cross section that we have estimated. Its inclusion in the rate equations, however, does not make a discernible difference for all higher charge species and, in particular, for the discussion on the $\mathrm{Ar}^{7+}$ production. This can be attributed to the fact that the chain of single-photon, single-electron processes leading to $\mathrm{Ar}^{3+}$ overwhelm the direct $2 e$ process, so that a $20 \%$ difference in the yield of $\mathrm{Ar}^{3+}$ is hardly visible in the experimental data.

In the interest of exhaustive scrutiny of all conceivable influences at play, we have also explored the possibility of second harmonic contamination of the radiation. In view of the precautions taken in the experimental setup, especially the strong suppression of this wavelength by the extremely small reflectivity of the multilayer mirror, we have concluded that the conceivable amount of second harmonic in the radiation was by at least 2 orders of magnitude too small to influence the observed $\mathrm{Ar}^{7+}$ yield.

\section{RESULTS AND DISCUSSION}

A typical ion time-of-flight spectrum of Ar obtained at maximal FEL pulse energy is shown in Fig. 1. Charge states up to $\mathrm{Ar}^{7+}$ are clearly observable. Principally, the creation of high ionic states in the soft-X-ray regime is understood

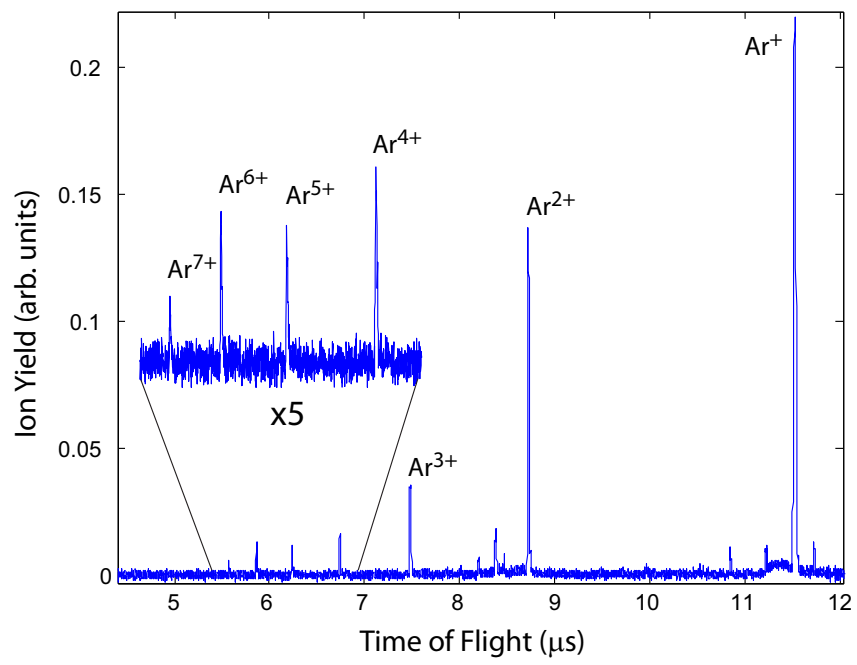

FIG. 1. Typical time-of-flight spectrum of atomic Ar irradiated with intense FEL radiation of $105 \mathrm{eV}$ photon energy and pulse energies of $16 \mu \mathrm{J}$ in the interaction region. The small, nonlabeled contributions in the spectrum are caused by the subsequent FEL pulse as well as by ionization of the residual gas in the experimental chamber. 


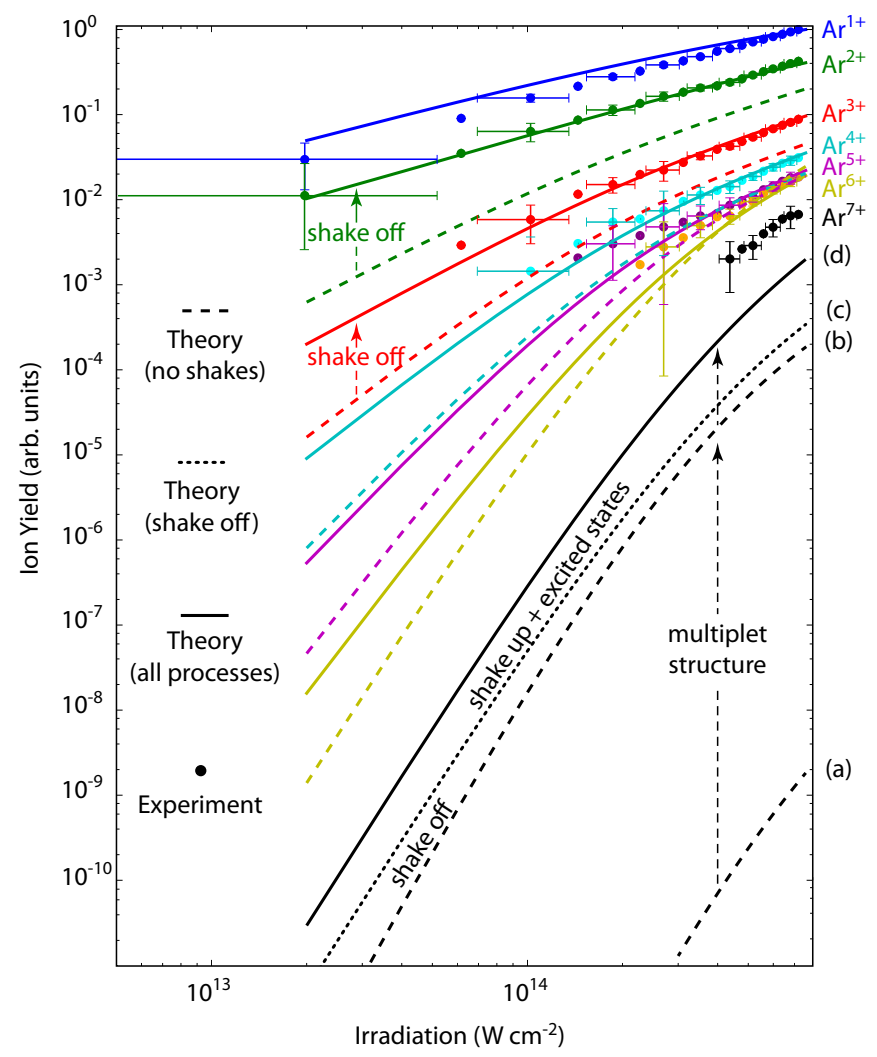

FIG. 2. Comparison of experimental (symbols) and theoretical results [model including (solid lines) and excluding (dashed lines) shake processes] of the relative ionic charge state distribution of atomic Ar upon radiation with intense FEL pulses of $105 \mathrm{eV}$ photon energy. Experimental and theoretical curves are normalized to a relative yield of 1 for $\mathrm{Ar}^{+}$at the highest FEL intensity. For the $\mathrm{Ar}^{7+}$ yield the results of the different theoretical models are indicated: (a) including only the electronic ground state, (b) including the $\operatorname{Ar}^{7+}(3 s 3 p)^{2}$ multiplet structure, (c) adding shake-off processes (dotted line), and (d) adding shake-up processes.

to predominantly be a result of a multiple photon-matter interaction within a very short time. For radiation intensities so far achieved at state-of-the-art FELs, the cross section of sequential multiphoton absorption is calculated to be much higher than the cross section of simultaneous photon absorption [8-10,23]. Regarding sequential ionization, it is commonly assumed that after one ionization step the target relaxes in the respective ionic ground state. This ionic ground state then forms a new target which, e.g., in case of a highly intense FEL pulse, is further ionized within the same photon pulse. Depending on the interaction probability, these steps of ionization are repeated until the FEL pulse is over and/or the target cannot be further ionized by the respective photon energy. For a photon energy of $105 \mathrm{eV}$, neutral argon and ionic species up to and including $\mathrm{Ar}^{5+}$ are predominantly ionized through these sequential single-photon absorption processes.

The dependence of the individual charge states on the pulse energy of the FEL radiation is displayed in Fig. 2 in the range from $2 \times 10^{13}$ to $7 \times 10^{14} \mathrm{~W} \mathrm{~cm}^{-2}$. The experimental data (data points with error bars) are compared to the results of our calculations using different approximations (dashed and solid
TABLE I. Relative yields for the different ion states of atomic Ar after irradiation with XUV pulses of $105 \mathrm{eV}$ photon energy and pulse intensities of $7.2 \times 10^{14} \mathrm{~W} \mathrm{~cm}^{-2}$. For the experiment the statistical errors are indicated in parentheses (errors related to FEL pulse intensity are given in Fig. 2). The columns for the theoretical results represent different calculations including (column 3) or not including (column 4) shake-up and shake-off.

\begin{tabular}{|c|c|c|c|}
\hline \multirow[t]{2}{*}{ Ionic state } & \multirow[t]{2}{*}{ Experiment } & \multicolumn{2}{|c|}{ Theory } \\
\hline & & $\begin{array}{c}\text { Shake-up + } \\
\text { shake-off }\end{array}$ & No shake \\
\hline $\mathrm{Ar}^{+}$ & $1.00(2)$ & 1 & 1 \\
\hline $\mathrm{Ar}^{2+}$ & $0.42(2)$ & 0.41 & 0.20 \\
\hline $\mathrm{Ar}^{3+}$ & $8.8(7) \times 10^{-2}$ & $9.6 \times 10^{-2}$ & $4.5 \times 10^{-2}$ \\
\hline $\mathrm{Ar}^{4+}$ & $3.1(6) \times 10^{-2}$ & $3.5 \times 10^{-2}$ & $2.0 \times 10^{-2}$ \\
\hline $\mathrm{Ar}^{5+}$ & $1.8(5) \times 10^{-2}$ & $2.2 \times 10^{-2}$ & $1.9 \times 10^{-2}$ \\
\hline $\mathrm{Ar}^{6+}$ & $1.9(5) \times 10^{-2}$ & $1.9 \times 10^{-2}$ & $2.4 \times 10^{-2}$ \\
\hline $\mathbf{A} \mathbf{r}^{7+}$ & $0.7(3) \times 10^{-2}$ & $0.2 \times 10^{-2}$ & $0.019 \times 10^{-2}$ \\
\hline
\end{tabular}

lines). In solving the rate equations, we considered pulses with Gaussian temporal profiles, peak intensities in the range of $10^{13}-10^{16} \mathrm{~W} \mathrm{~cm}{ }^{-2}$, and a pulse duration of $80 \mathrm{fs}$. Given that we are dealing with single-photon processes, with only one twophoton process involved in the last step of the observed ionic species, the usual intensity fluctuations inherent in FEL pulses, produced by self-amplified stimulated emission (SASE), are of negligible importance for the resulting ionic yields. Therefore, employing a Gaussian temporal profile is well justified. The simplest, as well as dominant, sequence of channels in the process of multiple ionization under these conditions, consists of those that leave all successive ions in the ground state. These are referred to hereafter as the primary channels.

Calculations, which included only the primary channels, produced ionic species populations in only qualitative overall agreement with the experimental data. For the low charge states, especially $\mathrm{Ar}^{2+}$ to $\mathrm{Ar}^{4+}$, the calculated yields differ by a factor of 2 and for $\mathrm{Ar}^{7+}$ even by more than 1 order of magnitude (Table I). Since ionization from the $\mathrm{Ar}^{6+} 3 s^{2}$ ground state requires a photon energy of more than $124.4 \mathrm{eV}$, one possibility for production of $\mathrm{Ar}^{7+}$ is given via two-photon ionization of one $3 s$ electron. With the calculated value of $10^{-54} \mathrm{~cm}^{4} \mathrm{~s}$ for the respective two-photon ionization cross section, the resulting $\mathrm{Ar}^{7+}$ yield turns out to be 6 orders of magnitude smaller than observed. The possible uncertainty of perhaps a factor of 2 or so in the two-photon cross section obviously is by far too small to bridge the theory versus experiment gap. Moreover, the possibility of enhancement from an intermediate single-photon resonance can be ruled out. The nearest resonance is detuned by $2.5 \mathrm{eV}$ and the FEL bandwidth is too narrow, while ac Stark shifts are found to be way too small to cause a transient resonance of any type [11,24-26]. There is, however, one excited state of $\mathrm{Ar}^{6+}$, namely $3 s 3 p\left({ }^{1} P_{1}\right)$, lying $21.2 \mathrm{eV}$ above the ground state $3 s^{2}$, which is created through the single-photon sequence of transitions $\operatorname{Ar}^{4+}\left(3 s^{2} 3 p^{2}\right) \rightarrow$ $\mathrm{Ar}^{5+}\left(3 s 3 p^{2}\right) \rightarrow \mathrm{Ar}^{6+} 3 s 3 p$. Single-photon ionization of the $3 p$ electron of that state accounts for the principal portion of the $\mathrm{Ar}^{7+}$ yield, reducing the discrepancy between experiment and theory from a factor of $10^{6}$ to a factor of about 40 (cf. 
Table I). The example illustrates the strong sensitivity of the calculations to the proper treatment of the involved electron configurations for certain photon energies, but demonstrates also that additional refinements are still necessary to further reduce the discrepancies to the experiment.

We consider now the possible role of electron correlations which manifest themselves in shake-up and shake-off processes leading to the formation of excited states or the simultaneous ejection of two electrons, respectively. In the sequence of ionization, although ionization of the excited ionic states might be expected to contribute to the creation of the next species, their contribution is expected to be small. However, inclusion of these processes leads to a reasonable overall agreement for the charge states up to and including $\mathrm{Ar}^{6+}$. Since normalization of the experimental and theoretical data is done on the singly charged ion, which yields only contributions from shake-up processes, the influence of shake-off shows up clearly in the yields of $\mathrm{Ar}^{2+}$ and $\mathrm{Ar}^{3+}$ (Fig. 2). The relative importance of both processes can be evaluated for the ionization step $\mathrm{Ar}^{6+} \rightarrow \mathrm{Ar}^{7+}$. For photon energy of $105 \mathrm{eV}$, the ejection of one electron from the $3 s^{2}$ ground state of $\mathrm{Ar}^{6+}$ is a two-photon process, while ionization of the highest $3 s 3 p$ multiplet state or the ejection of a $3 d$ or another outer electron from an excited state [e.g., $\operatorname{Ar}^{6+}(3 s 3 d)$ ] is a single-photon process. Upon quantitative analysis, it turns out that the contribution of the latter, at the intensities and pulse duration of the experiment, is several orders of magnitude larger than that of the two-photon process, although the population of $\operatorname{Ar}^{6+}(3 s 3 d)$ never amounts to more than $1 \%$ of the population of $\mathrm{Ar}^{6+}\left(3 s^{2}\right)$. After initial formation of the excited state through electron correlation in the first step of the ionization sequence, e.g., $\mathrm{Ar}^{+} 3 p^{4} 3 d$, a significant percentage of the excited electron population survives during the subsequent ionization processes, since the cross section for ionization of the $3 d$ electron in the excited singly charged ion is considerably smaller (by almost 1 order of magnitude) than the cross section for ionization in the $3 p$ shell. However, for higher ionic species, an electron in the same excited $3 d$ state is more strongly bound, which endows it with a larger ionization cross section, becoming eventually equal to the $3 p$ ionization for $\mathrm{Ar}^{5+}$. We have thus the surprising situation in which, through the interplay of linear with nonlinear processes, a traditionally small effect exerts a disproportionally large influence.

For neutral Ar, it is known [1,27] that for photons of $105 \mathrm{eV}$, there are channels leaving the ion predominantly in the $3 d$ state, with much smaller percentages going to the $4 d$ and $5 d$ states. The relevant cross section is about 0.02 $\mathrm{Mb}$, whereas the cross section for the primary channel is essentially $1 \mathrm{Mb}$, confirming that ionization plus excitation per fine structure pair of channels amounts to a few percent of the primary process. In a more recent paper on neon [28], considerably larger contributions of shake-up processes have been noted. To the best of our knowledge, nothing is known about the corresponding cross sections for the ions, which implied we should proceed on the basis of some estimates. First, all contributions from excited states are taken into account by considering the $3 d$ state. Second, the cross section for ionization plus excitation should be smaller than that for the primary channel. Other than that, we have explored values from about $10 \%$ to $50 \%$. Although, in principle, this ratio would depend on the ionic species, in view of the large number of channels involved in each step, the overall (effective) percentage might not vary too much. In the final calculations, we account in each step for the ionization of the excited state in the parent ion via ejection of the $3 d, 3 s$, or $3 p$ electron and the creation of additional $3 d$ electrons (through the process of ionization with excitation described above) in the daughter ion. This chain of channels leads to a population of $\mathrm{Ar}^{6+}(3 s 3 d)$, which is much smaller than that of $\mathrm{Ar}^{6+}\left(3 s^{2}\right)$. We have calculated the ionization cross sections of the $3 d$ states of all ionic species. Inserting all of the above channels into an expanded set of rate equations, we obtained the results depicted in Fig. 2 (solid lines).

The population of $\mathrm{Ar}^{7+}$ resulting from this set of equations agrees now to within a factor of 3.5 with the experimental data (see Table I). To obtain this agreement, we had to assume ionization plus excitation cross sections of about $50 \%$ of the primary channels. The $\mathrm{Ar}^{7+}$ yield is now about 5 orders of magnitude larger than it was without the inclusion of excited states and of two-electron processes (see Fig. 2). On the basis of single-photon physics, this result is counterintuitive, because under single-photon ionization, another single-photon channel, with a cross section equal to a fraction of that of the primary channel, will simply make a contribution equal to that fraction. However, what we have here is an entirely different situation; because although the population of the excited state in $\mathrm{Ar}^{6+}$ is only a percentage of that in the ground state, the latter can be ionized only by two-photon absorption. As a result, the lower order of nonlinearity in the ionization of the excited state more than makes up for the smaller population in that state. A salient point here is that, owing to the tighter binding of the orbitals for the higher ionic species, the ionization cross section of the excited electrons increases with increasing charge state, contributing thus to the unexpectedly large effectiveness of this channel in the last ionization step. Thus although part of the excited states in each species is ionized in the next step, still the balance between excitation and loss of the excited state through ionization is such as to leave population in the effective excited state.

The important role of the excitation channel is clearly illustrated in Table I, which shows the relative yields of all charge states for the highest FEL intensities $\left(7.2 \times 10^{14}\right.$ $\mathrm{W} \mathrm{cm}^{-2}$ ). The comparison of the experimental values with the results of the different theoretical models demonstrates the significant role of shake processes for the quantitative understanding of the observed multiple ionization processes. The relative strength of the yields for the different charge states, in particular the strong population of $\mathrm{Ar}^{7+}$ observed in the experiment, cannot be accounted for without the inclusion of two-electron processes, i.e., shake-up and shakeoff processes.

\section{CONCLUSION}

This work has uncovered two-electron processes hidden in the ion data that normally are insensitive to such processes. Beyond the interpretation of the most prominent features of the ionic yields in this work, we have uncovered a number of open problems, such as ionization plus excitation in ionic species. Although studies using synchrotron radiation have provided 
data for those processes in the neutral, their counterpart for ions are, to the best of our knowledge, unknown territory. Clearly, the abundant production of ionic species under FEL radiation provides a unique tool for their study, with minimal experimental requirements relying simply on the FEL intensity dependence of ionic yields. Obviously, more refined information can be expected and is in fact needed if photoelectron energy spectra are included in the measurements. Another surprise was the detection of the role of single-photon double ionization, in the same context, which normally requires a more demanding experimental arrangement. The results of this work suggest a hitherto unsuspected richness in multiple ionization under FEL radiation, pointing to possibly the need for the re-examination of previous and future work, on other atomic and of course molecular species, where otherwise unavailable information, such as shake-up processes in ionic species, is most likely hidden.

Having summarized our findings, we would like to underscore an apparent puzzle in the comparison of theory with the experimental data. On the one hand, even within the estimated uncertainties of our cross sections, definitive signatures of shake-off processes are documented in the yields of the lower ionic species. On the other hand, the reason for the remaining discrepancy of about a factor of 3.5 for $\mathrm{Ar}^{7+}$ is at this stage impossible to pinpoint. To the best of our knowledge, all significant channels to that species have been taken into consideration. Yet, even with admittedly somewhat large shake-up cross sections, the theoretical yield remains below the experimental data. It would take an unphysically gigantic two-photon cross section to account for that discrepancy. Such a two-photon cross section can be ruled out with certainty. We are thus compelled to accept that puzzle, leaving its solution to future work.

\section{ACKNOWLEDGMENTS}

We thank the DESY staff for providing smooth machine conditions of FLASH during the beam times. M.I., S.B., A.J.R., and N.G. are indebted to the SFB 925 Light Induced Dynamics and Control of Correlated Quantum Systems for financial support. M.I. is furthermore grateful for funding from the Volkswagen foundation. The DCU group acknowledges support by SFI Grant No. 12/IA/1742. The work was supported by the European CMST COST Action CM1204 (XLIC).

\section{APPENDIX}

The complete set of coupled rate equations describing the overall production of argon ions irrespective of atomic states and configurations in each ionic species, which is what is measured in the experiment, is given by

$$
\begin{aligned}
& \frac{d N_{0}}{d t}=-\left[\left(\sigma_{10}+\sigma_{10 \mathrm{exc}}+\sigma_{10 \mathrm{sh}}+\sigma_{20 \mathrm{so}}\right) F(t)+\left(\sigma_{\mathrm{ATI}+}^{(2)}+\sigma_{20}^{(2)}\right) F^{2}(t)\right] N_{0}, \\
& \frac{d N_{1}}{d t}=\left[\left(\sigma_{10}+\sigma_{10 \mathrm{exc}}+\sigma_{10 \mathrm{sh}}\right) F(t)+\sigma_{\mathrm{ATI}+}^{(2)} F^{2}(t)\right] N_{0}-\left[\left(\sigma_{21}+\sigma_{21 \mathrm{exc}}+\sigma_{21 d}+\sigma_{21 \mathrm{sh}}+\sigma_{31 \mathrm{so}}\right) F(t)+\sigma_{\mathrm{ATI} 2+}^{(2)} F^{2}(t)\right] N_{1}, \\
& \frac{d N_{2}}{d t}=\left[\sigma_{20 \mathrm{so}} F(t)+\sigma_{20}^{(2)} F^{2}(t)\right] N_{0}+\left[\left(\sigma_{21}+\sigma_{21 \mathrm{exc}}+\sigma_{21 d}+\sigma_{21 \mathrm{sh}}\right) F(t)+\sigma_{\mathrm{ATI} 2+}^{(2)} F^{2}(t)\right] N_{1}-\left(\sigma_{32}+\sigma_{32 \mathrm{exc}}+\sigma_{32 d}+\sigma_{32 \mathrm{sh}}\right) F(t) N_{2}, \\
& \frac{d N_{3}}{d t}=\sigma_{31 \mathrm{so}} F(t) N_{1}+\left(\sigma_{32}+\sigma_{32 \mathrm{exc}}+\sigma_{32 d}+\sigma_{32 \mathrm{sh}}\right) F(t) N_{2}-\left(\sigma_{43}+\sigma_{43 \mathrm{exc}}+\sigma_{43 d}+\sigma_{43 \mathrm{sh}}\right) F(t) N_{3}, \\
& \frac{d N_{4}}{d t}=\left(\sigma_{43}+\sigma_{43 \mathrm{exc}}+\sigma_{43 d}+\sigma_{43 \mathrm{sh}}\right) F(t) N_{3}-\left(\sigma_{54}+\sigma_{54 \mathrm{exc}}+\sigma_{54 d}+\sigma_{54 \mathrm{sh}}\right) F(t) N_{4}, \\
& \frac{d N_{5}}{d t}=\left(\sigma_{54}+\sigma_{54 \mathrm{exc}}+\sigma_{54 d}+\sigma_{54 \mathrm{sh}}\right) F(t) N_{4}-\left(\sigma_{65}+\sigma_{65 \mathrm{exc}}+\sigma_{65 d}+\sigma_{65 \mathrm{sh}}\right) F(t) N_{5}, \\
& \frac{d N_{6}}{d t}=\left(\sigma_{65}+\sigma_{65 \mathrm{exc}}+\sigma_{65 d}+\sigma_{65 \mathrm{sh}}\right) F(t) N_{5}-\left[\left(\sigma_{76 d}+\sigma_{76 \mathrm{sh}}\right) F(t)+\sigma_{76}^{(2)} F^{2}(t)+\sigma_{86}^{(3)} F^{3}(t)\right] N_{6}, \\
& \frac{d N_{7}}{d t}=\left[\left(\sigma_{76 d}+\sigma_{76 \mathrm{sh}}\right) F(t)+\sigma_{76}^{(2)} F^{2}(t)\right] N_{6}-\sigma_{87}^{(2)} F^{2}(t) N_{7}, \\
& \frac{d N_{8}}{d t}=\sigma_{86}^{(3)} F^{3}(t) N_{6}+\sigma_{87}^{(2)} F^{2}(t) N_{7},
\end{aligned}
$$

where $N_{i}$ denotes the population of an ion with charge $+i . \sigma_{i j}^{(n)}$ denotes the ionization cross section for ionization of an ion with charge $+j$ via an $n$-photon process thereby creating an ion with charge $+i$. The number of photons involved was omitted for single-photon processes in order to keep the notation as simple as possible. Other processes are denoted through additional indices, where exc stands for the process of simultaneous ionization of an electron while exciting another to a $d$-state orbital, $d$ denotes the ionization cross section of these excited $d$ states, $s h$ denotes ionization through radiation of the second harmonic, and so denotes the shake-off processes. The values of the cross sections are given in Table II.

The single-photon ionization cross sections except for $\sigma_{\text {exc }}$ were obtained using Cowan's code [29,30]. Given that we needed many single-photon cross sections, for several ionic species each with a different structure, for reasons of selfconsistency it was desirable to employ a method applicable to all. Cowan's code, which has been found quite adequate in such a context [31], offers the necessary flexibility for 
TABLE II. Values of single-photon, ATI, and multiphoton cross sections.

\begin{tabular}{|c|c|c|}
\hline \multicolumn{3}{|c|}{ Single photon cross sections } \\
\hline$\sigma_{10}=1.2 \times 10^{-18} \mathrm{~cm}^{2}$ & $\sigma_{21}=2.4 \times 10^{-18} \mathrm{~cm}^{2}$ & $\sigma_{32}=3.5 \times 10^{-18} \mathrm{~cm}^{2}$ \\
\hline$\sigma_{43}=3.3 \times 10^{-18} \mathrm{~cm}^{2}$ & $\sigma_{54}=2.9 \times 10^{-18} \mathrm{~cm}^{2}$ & $\sigma_{65}=1.8 \times 10^{-18} \mathrm{~cm}^{2}$ \\
\hline$\sigma_{10 \mathrm{sh}}=1.7 \times 10^{-24} \mathrm{~cm}^{2}$ & $\sigma_{21 \mathrm{sh}}=1.9 \times 10^{-24} \mathrm{~cm}^{2}$ & $\sigma_{32 \mathrm{sh}}=1.5 \times 10^{-24} \mathrm{~cm}^{2}$ \\
\hline$\sigma_{43 \mathrm{sh}}=1.7 \times 10^{-24} \mathrm{~cm}^{2}$ & $\sigma_{54 \mathrm{sh}}=1.4 \times 10^{-24} \mathrm{~cm}^{2}$ & $\sigma_{65 \mathrm{sh}}=1.0 \times 10^{-24} \mathrm{~cm}^{2}$ \\
\hline$\sigma_{76 \mathrm{sh}}=0.8 \times 10^{-24} \mathrm{~cm}^{2}$ & & \\
\hline$\sigma_{10 \mathrm{exc}}=4.5 \times 10^{-20} \mathrm{~cm}^{2}$ & $\sigma_{21 \mathrm{exc}}=4.4 \times 10^{-20} \mathrm{~cm}^{2}$ & $\sigma_{32 \mathrm{exc}}=4.0 \times 10^{-20} \mathrm{~cm}^{2}$ \\
\hline$\sigma_{43 \mathrm{exc}}=3.5 \times 10^{-20} \mathrm{~cm}^{2}$ & $\sigma_{54 \mathrm{exc}}=2.7 \times 10^{-20} \mathrm{~cm}^{2}$ & $\sigma_{65 \mathrm{exc}}=2.6 \times 10^{-20} \mathrm{~cm}^{2}$ \\
\hline$\sigma_{21 d}=5.0 \times 10^{-20} \mathrm{~cm}^{2}$ & $\sigma_{32 d}=1.3 \times 10^{-19} \mathrm{~cm}^{2}$ & $\sigma_{43 d}=1.0 \times 10^{-19} \mathrm{~cm}^{2}$ \\
\hline$\sigma_{54 d}=2.5 \times 10^{-19} \mathrm{~cm}^{2}$ & $\sigma_{65 d}=3.0 \times 10^{-19} \mathrm{~cm}^{2}$ & $\sigma_{76 d}=8.0 \times 10^{-19} \mathrm{~cm}^{2}$ \\
\hline$\sigma_{20 \mathrm{so}}=2.5 \times 10^{-19} \mathrm{~cm}^{2}$ & $\sigma_{31 \mathrm{so}}=8 \times 10^{-20} \mathrm{~cm}^{2}$ & \\
\hline \multicolumn{3}{|c|}{ ATI cross sections } \\
\hline$\sigma_{\mathrm{ATI}^{+}}^{(2)}=7 \times 10^{-53} \mathrm{~cm}^{4} \mathrm{~s}$ & $\sigma_{\mathrm{ATI}^{2+}}^{(2)}=3 \times 10^{-53} \mathrm{~cm}^{4} \mathrm{~s}$ & \\
\hline \multicolumn{3}{|c|}{ Multiphoton cross sections } \\
\hline $\begin{array}{l}\sigma_{20}^{(2)}=6 \times 10^{-54} \mathrm{~cm}^{4} \mathrm{~s} \\
\sigma_{86}^{(3)}=1 \times 10^{-90} \mathrm{~cm}^{6} \mathrm{~s}^{2}\end{array}$ & $\sigma_{76}^{(2)}=2.9 \times 10^{-56} \mathrm{~cm}^{4} \mathrm{~s}$ & $\sigma_{87}^{(2)}=6.6 \times 10^{-54} \mathrm{~cm}^{4} \mathrm{~s}$ \\
\hline
\end{tabular}

the task. The two-photon cross sections $\sigma_{76}^{(2)}$ and $\sigma_{87}^{(2)}$ were calculated with the single-channel quantum defect theory. Its accuracy was tested by trial calculations for systems for which values of cross sections obtained through other methods were available. Finally, the value of the single-photon cross sections for shake-up and shake-off in the neutral was obtained from the literature, which allowed us to estimate the corresponding values for the ion by extrapolation.
[1] W. Wijesundera and H. P. Kelly, Phys. Rev. A 36, 4539 (1987).

[2] A. Starace and S. Manson, in VUV and Soft X-Ray Photoionization, edited by U. Becker and D. A. Shirley (Plenum, New York, 1996).

[3] J. Briggs and V. Schmidt, J. Phys. B 33, R1 (2000).

[4] H. Kossmann, B. Krässig, V. Schmidt, and J. E. Hansen, Phys. Rev. Lett. 58, 1620 (1987).

[5] U. Becker and D. Shirley, Phys. Scr., T31, 56 (1990).

[6] A. A. Sorokin, S. V. Bobashev, T. Feigl, K. Tiedtke, H. Wabnitz, and M. Richter, Phys. Rev. Lett. 99, 213002 (2007).

[7] M. Richter, M. Y. Amusia, S. V. Bobashev, T. Feigl, P. N. Juranic, M. Martins, A. A. Sorokin, and K. Tiedtke, Phys. Rev. Lett. 102, 163002 (2009).

[8] N. Berrah et al., J. Mod. Opt. 57, 1015 (2010).

[9] L. Young et al., Nature (London) 466, 56 (2010).

[10] P. Lambropoulos, K. G. Papamihail, and P. Decleva, J. Phys. B: At. Mol. Opt. Phys. 44, 175402 (2011).

[11] B. Rudek et al., Nat. Photonics 6, 858 (2012).

[12] H. Fukuzawa, S.-K. Son, K. Motomura, S. Mondal, K. Nagaya, S. Wada, X.-J. Liu, R. Feifel, T. Tachibana, Y. Ito, M. Kimura, T. Sakai, K. Matsunami, H. Hayashita, J. Kajikawa, P. Johnsson, M. Siano, E. Kukk, B. Rudek, B. Erk, L. Foucar, E. Robert, C. Miron, K. Tono, Y. Inubushi, T. Hatsui, M. Yabashi, M. Yao, R. Santra, and K. Ueda, Phys. Rev. Lett. 110, 173005 (2013).

[13] P. J. Ho, C. Bostedt, S. Schorb, and L. Young, Phys. Rev. Lett. 113, 253001 (2014).

[14] R. Wehlitz, F. Heiser, O. Hemmers, B. Langer, A. Menzel, and U. Becker, Phys. Rev. Lett. 67, 3764 (1991).

[15] K.-H. Schartner et al., Phys. Scr. 41, 853 (1990).

[16] L. J. Frasinski et al., Phys. Rev. Lett. 111, 073002 (2013).

[17] K. Tiedtke et al., New J. Phys. 11, 023029 (2009).
[18] Y. Inubushi, K. Tono, T. Togashi, T. Sato, T. Hatsui, T. Kameshima, K. Togawa, T. Hara, T. Tanaka, H. Tanaka, T. Ishikawa, and M. Yabashi, Phys. Rev. Lett. 109, 144801 (2012).

[19] K. Tiedtke et al., J. Appl. Phys. 103, 094511 (2008).

[20] V. Richardson, J. T. Costello, D. Cubaynes, S. Dusterer, J. Feldhaus, H. W. van der Hart, P. Juranić, W. B. Li, M. Meyer, M. Richter, A. A. Sorokin, and K. Tiedke, Phys. Rev. Lett. 105, 013001 (2010).

[21] M. Meyer, D. Cubaynes, V. Richardson, J. T. Costello, P. Radcliffe, W. B. Li, S. Dusterer, S. Fritzsche, A. Mihelic, K. G. Papamihail, and P. Lambropoulos, Phys. Rev. Lett. 104, 213001 (2010).

[22] T. Mazza et al., Nat. Commun. 6, 6799 (2015).

[23] P. Lambropoulos, L. A. A. Nikolopoulos, M. G. Makris, and A. Mihelic, Phys. Rev. A 78, 055402 (2008).

[24] R. R. Freeman, P. H. Bucksbaum, H. Milchberg, S. Darack, D. Schumacher, and M. E. Geusic, Phys. Rev. Lett. 59, 1092 (1987).

[25] X. Tang, A. Lyras, and P. Lambropoulos, Phys. Rev. Lett. 63, 972 (1989).

[26] S. N. Dixit and P. Lambropoulos, Phys. Rev. A 27, 861 (1983).

[27] A. Kikas et al., J. Electron Spectrosc. Relat. Phenom. 77, 241 (1996).

[28] J. Feist, O. Zatsarinny, S. Nagele, R. Pazourek, J. Burgdorfer, X. Guan, K. Bartschat, and B. I. Schneider, Phys. Rev. A 89, 033417 (2014).

[29] R. D. Cowan, http://aphysics2.lanl.gov/cgi-bin/ION/ runlanl08d.pl.

[30] R. D. Cowan, The Theory of Atomic Structure and Spectra (University of California, Berkeley, 1981).

[31] G. M. Nikolopoulos and P. Lambropoulos, J. Phys. B 47, 115001 (2014). 